\title{
Is there a place for corticosteroids or immune modulation in treating Reiter's syndrome and ankylosing spondylitis?
}

\author{
M. ROSENTHAL \\ From the Merian Iselin Hospital, Basel, Switzerland
}

\begin{abstract}
Most patients with Reiter's syndrome (RS) or B27associated conditions respond well to non-steroidal anti-inflammatory agents. Hence there is no need for corticosteroid therapy or immune modulation in mild cases. Aggressive treatment should be considered only if a patient develops severe systemic manifestations such as vasculitis or iridocyclitis or the disease has a prolonged, crippling course that does not respond to conventional therapy.
\end{abstract}

\section{Corticosteroids}

Severe cases of mono- or oligoarthritis usually respond very well to intra-articular corticosteroid applications, which often allow a reduction in the daily dose of anti-inflammatory agents. Moreover, in other manifestations such as eye lesions which require corticosteroids local administration is generally preferable.

Corticosteroids seem less effective in RS and ankylosing spondylitis (AS) than in other connective tissue diseases. Also they may promote infections, especially of an agent causing the disease. Thus systemic corticosteroid treatment should be given only when other treatments fail. In such cases the initial dose should be high $(0 \cdot 5-1$ $\mathrm{mg} / \mathrm{kg}$ body weight prednisone or equivalent) gradually reducing to the lowest dose that controls symptoms.

\section{Gold salts}

Gold salts have also been given for RS, especially in cases where polyarthritis is the most pronounced symptom. The effectiveness of this empirical treatment has never been proved and it should therefore not be used.

\section{Cytostatic and immunosuppressive agents}

Severe, progressive cases of RS and AS uncontrolled by either anti-inflammatory agents or corticosteroids have been given immunosuppressive treatment, mostly with azathioprine. In most cases disease activity was significantly reduced. The treatment is hazardous and requires close monitoring.

\section{Levamisole}

There have been several reports on the treatment of RS and AS with levamisole, an antihelminthic drug with immunorestorative capacities. ${ }^{9,128,162,261}$ This preparation proved very effective in rheumatoid arthritis. We found that it induced a significant improvement in seven out of 10 patients with RS and eight out of 15 patients with AS. Allen ${ }^{9}$ and Ippen ${ }^{162}$ and their colleagues, in uncontrolled open studies, reported a few patients with RS who all improved dramatically on levamisole.

By far the most extensive study of the effect of levamisole in HLA-B27-associated diseases was by Goebel et al. ${ }^{128}$ They studied 37 men with seronegative HLA-B27-positive spondyloarthritis, including 18 classical cases of AS, 14 cases of RS, and five cases of psoriatic arthritis in a randomised cross-over trial over a period of 12 weeks. There was a significant clinical improvement in the levamisole group compared with placebo, and also some laboratory parameters showed significant improvement. Out of about 50 levamisole-treated cases of RS $15(30 \%)$ showed adverse reactions, mostly mild, including maculopapular rash, nausea and vomiting, and leucopenia. Two patients developed agranulocytosis but recovered spontaneously within a few days. My experience of levamisole so far is limited to giving it to 20 patients with 'drug refractory' RS. Fifteen of them distinctly improved. Out of 15 patients with AS the results were encouraging in eight (Table).

It seems that levamisole can benefit severe cases of RS, but extensive studies are still required to establish this. Levamisole seems more effective in treating oligo- and polyarthritis; less effective in improving spondylitis, stomatitis, and urethritis; 
Table Apparent clinical effect of levamisole in 20 cases of Reiter's syndrome and 15 cases of ankylosing spondylitis

\begin{tabular}{lcc}
\hline Clinical features & No. of cases & $\begin{array}{c}\text { Response to } \\
\text { levamisole }\end{array}$ \\
\hline Polyarthritis & 22 & + \\
Oligoarthritis & 13 & + \\
Spondylitis & 15 & \pm \\
Stomatitis & 12 & \pm \\
Urethritis/balanitis & 10 & \pm \\
Iritis/conjunctivitis & 7 & - \\
\multicolumn{1}{c}{ Acute cases } & 9 & \pm \\
Chronic cases & 26 & + \\
\hline
\end{tabular}

*Effective $=+$. Occasionally effective $= \pm$. No effect $=-$.

and ineffective in iritis and conjunctivitis. Acute cases seem to respond less than chronic cases.

\section{General discussion}

A SPEAKER: What is the rationale of using immune stimulating drugs in RS? Is there any proof of immune deficiency in this disease?

DR. ROSENTHAL: The same question arose for rheumatoid arthritis. A number of recent reports describe both humoral and cellular impairments in AS and in RS. We could see immune complex formation. Lymphocyte reaction to mitogens* may be impaired. Moreover, when used in the treatment of infectious diseases levamisole appears to improve defensive reactions of the host such as phagocytosis of bacteria. It was tried empirically in RS on the reasoning that RS might also be an infectious disease. It turned out to be of some clinical benefit. PROF. M. ZIFF: Is it really acting as an immunostimulatory drug or is it an anti-inflammatory agent?

DR. ROSENTHAL: We have shown in adjuvant disease of the rat that this experimental inflammatory disease actually became worse with levamisole. Had it any anti-inflammatory or immunosuppressive properties it should be beneficial to the animal and

*Mostly plant lectins such as phytohaemagglutinin, concanavalin A, pokeweed mitogen, etc. not enhance the disease, with increased destruction of joints.

DR. K. BANDILLA: It is said that B27-positive patients with rheumatoid arthritis treated with levamisole were prone to agranulocytosis. Isn't it risky to give the drug in diseases with a high incidence of B27positive patients?

DR. ROSENTHAL: Agranulocytosis is a not infrequent complication of any aggressive treatment in rheumatic diseases and cannot simply be attributed to B27. Eric Veys reviewed all the cases of agranulocytosis after levamisole treatment in RA and found that $80 \%$ of RA patients with this complication were rheumatoid factor-positive females carrying the phenotype B27. I would expect that out of this very special immunological constellation of females with rheumatoid factor-positive rheumatoid arthritis the B27 phenotype alone does not predispose to agranulocytosis. We encountered this complication in two patients with RS or AS treated with levamisole.

DR. T. M. SCOTT: You reviewed some work showing significant changes in immunoglobulin concentrations in patients treated with levamisole. Were these abnormal concentrations returning to normal or were they within the normal range throughout?

DR. ROSENTHAL: Those were not my data. Only the immunoglobulin- $M$ was higher than normal and it returned to normal after levamisole treatment. The other immunoglobulins were within normal range throughout.

DR. E. ALBERT: Do you consider levamisole as an immune potentiating agent?

DR. ROSENTHAL: It seems to be an immune restorative agent. I would not go beyond that.

DR. ALBERT: In which compartment does it restore immune reactivity? There is always the suspicion that stimulation in one compartment might be linked with immunosuppression in another.

DR. ROSENTHAL: This matter will be reviewed in the near future.* So far it seems that its main effect is to restore the suppressor cell effect when this had been deficient.

*Symposium on levamisole in Brussels, 7 June 1978. Journal of Rheumatology (Supplement 4), 5, 1978. 